\title{
“...RUTA DE FORMACIÓN PARA EL EMPRENDIMIENTO EN EDUCACIÓN BÁSICA PRIMARIA, SECUNDARIA Y MEDIA TÉCNICA..." EN COLOMBIA
}

\author{
Germán E. Lobo Rubio* \\ Carmen Aydé Rincón Becerra* \\ Luis Manuel Palomino Méndez ${ }^{* * *}$
}

\footnotetext{
* Docente Facultad de Ciencias Económicas y Empresariales Universidad de Pamplona. Pamplona Colombia. E-mail: globo@unipamplona.edu.co

** Docente Facultad de Ciencias Económicas y Empresariales Universidad de Pamplona. Pamplona Colombia. E-mail aydeerincon@unipamplona.edu.co

*** Docente Facultad de Ciencias Económicas y Empresariales Universidad de Pamplona. Pamplona Colombia. E-mail: luismanuel@unipamplona.edu.co
} 


\section{“...RUTA DE FORMACIÓN PARA EL EMPRENDIMIENTO EN EDUCACIÓN BÁSICA PRIMARIA, SECUNDARIA Y MEDIA TÉCNICA..." EN COLOMBIA}

\section{RESUMEN}

El presente artículo presenta las conclusiones del proyecto de investigación titulado: "El desarrollo de competencias emprendedoras básicas y transversales y su relación con el fomento a la cultura del emprendimiento y la generación de empresas exitosas" el cual fue propuesto para evidenciar soluciones a situaciones problemáticas detectadas en el ámbito académico relacionados con los procesos que orientan la formación para el emprendimiento y la praxis de procesos emprendedores.

El proyecto tenía por objetivo: Determinar los elementos que intervienen en la construcción de los estándares Básicos para el desarrollo de Competencias emprendedoras; que deben ser incluidos en los procesos de formación integral para el emprendimiento, desde la educación básica hasta la educación superior. Dicho proyecto obedece a la necesidad de crear herramientas que orienten los procesos de formación para el emprendimiento obligatoria ${ }^{1}$ en las instituciones públicas y privadas de básica primaria, secundaria y media técnica del país

Como resultado de la investigación se desarrollo la "Ruta de formación para el emprendimiento" que puede ser utilizada por las instituciones educativas $^{2}$ para optimizar los procesos de formación integral de los estudiantes, mediante el desarrollo de competencias emprendedoras básicas y transversales que maximicen sus características, valores y capacidades como emprendedores.

Palabras claves: Cultura del emprendimiento, competencia, ruta de formación, emprendedor, capital humano.

\begin{abstract}
This research article contains the investigation results of the project titled: "The development of basic enterprising competences and its cross relationship with the promotion to the entrepreneurial spirit culture and the generation of successful businesses". The purpose of this proposal was to show solutions to problem situations that are in the academic environment related to the processes that originated from the training and the practice of enterprising processes.

The objective of the project was: "Determining the elements that take part in the constructions of the basic standards for the development of enterprising competences that should be included in the processes of integral formation for to the enterprising since the basic education to the higher.

The project obeys to the need of tools that orient the processes of formation of the enterprising that is obligatory in public and private institutions of primary, sewndary and technical average in the country.

As result of the investigation developed in the formation route for the enterprising that can be used for the schools to optimize the (formation processes for the enterprising.) Training integral processes using the development of the basic and transversal enterprising competences to maximize their features, values and capacities entrepreneurships.
\end{abstract}

Key words: Enterprising culture, Competence, Training Route, Entrepreneurships, Human Capital.

\footnotetext{
1 Artículo 13 de la ley 1014 de 2006.de Fomento a la Cultura del Emprendimiento.

${ }^{2}$ Instituciones públicas y privadas de básica primaria, secundaria y media técnica del país.
}

\section{Face IsSN 1794-9920}

Recepción: Abril de 2008

Revisión: Mayo de 2008

Aceptación: Mayo de 2008 


\section{INTRODUCCIÓN}

Colombia en la actualidad hace grandes esfuerzos por promover la cultura del emprendimiento como alternativa para incentivar la creación de empresas, la generación de empleo y la de promover el desarrollo socioeconómico; razón por la cual se han implementado en los últimos años políticas gubernamentales, planes y programas encaminados a la creación de empresas y fomento del espíritu empresarial; apoyando a los emprendedores, facilitando su acceso al capital semilla o promoviendo procesos de formación, que le permitan el desarrollo de competencias laborales especificas; Dichas estrategias parecen estar mostrando algunos resultados en el mediano plazo.

Sin embargo algunos análisis más profundos le han permitido al gobierno nacional prever que se debían desarrollar nuevas acciones tendientes, no solo a proveer de herramientas a los jóvenes emprendedores en edad productiva capacitándolos o permitiéndoles el acceso a capital semilla sino que además, es igualmente importante incluir en los proceso de formación integral del capital humano en Colombia, estrategias de formación continuada que aseguraren a largo plazo desarrollar una verdadera cultura emprendedora en el país mediante la llamada institucionalización de la formación para el emprendimiento. Dicha institucionalización representa un gran avance legal sobre el tema, pero trae consigo problemas relacionados con los actores responsables y las herramientas disponibles para impartir formación para el emprendimiento, ya que si los actores no están preparados y no tienen las herramientas pues les será imposible cumplir con las responsabilidades asignadas.

Las situaciones anteriormente enunciadas dejan ver la importancia el desarrollo de propuestas investigativas tendientes a evaluar el papel de los actores (Instituciones Educativas) en cuanto a la capacidad técnica y operativa disponible requerida para llevar a cabo los procesos de formación para el emprendimiento, así como el desarrollo de propuestas metodológicas que orienten el trabajo de dichos actores.

El desarrollo social es en gran parte debido a la acción de tres fuerzas impulsoras que son: el gobierno, la empresa y la institución de educación (IE), por ello deben actuar en consenso y ayudarse mutuamente, la institución de educación (IE), por su parte, es la institución de la comunidad dedicada a la conquista del conocimiento, el estudio de la solución de sus problemas, la apreciación critica de las realizaciones y la formación de hombres a un alto nivel cultural, científico, ético y técnico. Su misión la cumple a través de una trilogía de funciones: Docencia, investigación, y proyección, tan entrelazadas entre sí, que para poder cumplir a cabalidad uno solo de sus objetivos, es necesario cumplir los otros dos y llegar a un concepto integral de trinidad. La investigación es básica para enriquecer los conocimientos y la docencia, el servicio, práctica o extensión, para probar la realidad de dichos conocimientos y para volver la docencia más relevante 
y mejorar la comunidad donde opera; la docencia, para impartir los conocimientos que vienen de tiempos anteriores, mejorados por la investigación y que el servicio ha puesto a prueba.

Con gran preocupación desde La Universidad de Pamplona se analizo tanto la situación de los actores, como la disponibilidad de herramientas didácticas y pedagógicas para impartir formación para el emprendimiento; por lo que se desarrollo del proyecto de investigación enfocado a "Determinar los elementos que intervienen en la construcción de los estándares Básicos para el desarrollo de Competencias emprendedoras; que deben ser incluidos en los procesos de formación integral para el emprendimiento, desde la educación básica hasta la educación superior".

El proyecto desarrollado por docentes adscritos a la Facultad de ciencias Económicas y Empresariales, de La Universidad de Pamplona. Surge a partir de un diagnostico que permitió determinar los aspectos a tenerse en cuenta en el desarrollo de herramientas que orienten el trabajo de los docentes encargados de las cátedras de emprendimiento en los Colegios; donde después de un análisis documental constructivo, se seleccionaron y propusieron las competencias para el emprendimiento discriminadas en las dimensiones del SER, SABER Y HACER que pueden ser desarrolladas por los estudiantes según el grado de escolaridad en el que se encuentre. Este diseño se denomino "Ruta de Formación Para el Emprendimiento" y presenta las unidades temáticas y contenidos a desarrollar en cada grado, cuidando de mantener una secuencia lógica en el desarrollo de los temas.

Es función básica de la institución de educación superior buscar y señalar caminos a sus alumnos y graduados, así como a la sociedad donde actúa y mostrarles sus obligaciones sociales y capacitarlos para dirigir a Colombia de cual son parte, dentro de la problemática social en que vivimos. Uno de los principales problemas es el de la pobreza, unida estrechamente con el desempleo y el subempleo; por ello una de las tareas más importantes es la de crear nuevas oportunidades de empleo remunerativo, es decir, nuevas empresas y mas riquezas y volver más eficientes a las actuales. Es importante lograr que cada empresa que exista en el país, sea pública o privada, grande, mediana o pequeña pueda ensancharse y crear más oportunidades. Con este concepto la enseñanza de la administración y el emprendimiento, cobra una importancia capital en todas las profesiones y niveles de educación. Si uno analiza cada posición en cualquier empresa u organización, así como la de cada individuo, ve claramente que si quiere progresar tiene que conocer al menos los principios fundamentales de la administración para manejar negocios propios o ajenos. Casi puede decirse que cuando una persona tiene éxito es porque ha llegado a un alto puesto administrativo. Por ello hay que propender para que los estudios del Emprendimiento y la administración se amplíen y penetren en otras carreras como las de ingenierías, tecnologías y licenciaturas y aun más en otros niveles de formación (básica primaria, secundaria y media técnica). 
La validación de la "Ruta de Formación Para el Emprendimiento" se inicio con la metodología denominada Taller investigativo que permitió después de la socialización de la misma a los docentes de cada Institución educativa, indagar acerca de la importancia y pertinencia de cada tema, objetando o proponiendo su inclusión. Esta herramienta es pues uno de los principales productos de esta investigación; sin embargo en el transcurso de la investigación también se evidencio que, la responsabilidad en la generación de jóvenes emprendedores, no es totalmente de las instituciones educativas debido a que los padres de familia tienen gran influencia en las primeras etapas de su vida en la formación de la personalidad (autoestima) de los jóvenes; factor clave para el desarrollo de un Emprendedor exitoso. Esta situación permitió, presentar como subproducto de la investigación, una propuesta de formación para padres orientada a responsabilizarles de su papel como "Formadores de Hombres y Mujeres Fracasados, mediocres o Emprendedores y Exitosos".

El proyecto concluye que para que pueda darse, la generación de una verdadera cultura del Emprendimiento es necesario Formar el capital Humano en Competencias propias del Emprendimiento, y no solo competencias laborales o empresariales ya que estas abarcan solo la dimensión del HACER, descuidando las Dimensiones del SER y del SABER de los emprendedores. Sin embargo es importante resaltar que para que a futuro se consolide una verdadera cultura del Emprendimiento es necesario continuar con el apoyo y fortalecimiento del Capital social, el Capital Institucional, y el Capital Financiero como lo plantean algunos teóricos en el tema de emprendimiento, pero además es absolutamente necesario responsabilizar a los padres de familia de su roll como formadores de emprendedores.

Tenemos que formar profesionales y dirigentes con una personalidad, unas actitudes y unas características que deben incluir las de: creatividad, innovación, capacidad investigativa, flexibilidad, deseo permanente de trabajar, aprender y perfeccionarse, independencia con responsabilidad social, deseo de logro, amor a la excelencia, capacidad de vencer el temor de enfrentar los riesgos, etc. Todo ello dentro de una formación integral, espiritual y ética.

Tenemos que comprender que la misión que debemos cumplir, especialmente en el campo administrativo, no es la de formar simples empleados, sino profesionales empresarios, que no se limiten a cumplir un oficio rutinario, si no que sean personas con capacidad de emprender acciones y crear entidades o mejorar las actuales con nuevas ideas que produzcan riqueza a la comunidad. 


\section{METODOLOGÍA (MATERIALES Y MÉTODOS)}

En el planteamiento de esta investigación se desarrollo desde un enfoque micro social de carácter cualitativo holístico, donde el objeto de estudio era el proceso de formación para el emprendimiento, desarrollado por las Instituciones Educativas de Básica Primaria, secundaria, y media Técnica en la ciudad de Pamplona las cuales se convirtieron en la muestra, y el universo serian todas las Instituciones educativas con las mismas características existentes en el país ya que guardan similitudes en estructura y desarrollo a excepción de las privadas ubicadas en grandes Capitales.

Entre los métodos utilizados para la elaboración de la propuesta metodológica de investigación, se destacaron, para la documentación inicial sobre la realidad específica de análisis, la exploración de la literatura y el "mapeo". Entre los instrumentos, estrategias, técnicas y medios para la generación y recolección de información, se utilizaron: el análisis documental, la encuesta etnográfica, el taller investigativo y finalmente como técnicas y medios para el análisis de la información se tuvieron en cuenta la apertura, la flexibilidad, sensibilidad estratégica y la referencialidad ${ }^{3}$.

\section{Resultados y discusión}

Como resultado del diagnostico inicial, se encontró que el $80 \%$ de los Colegios 4 encuestados, no están cumpliendo a la fecha (abril de 2007, un año después de promulgada la ley) con la inserción de la cátedra de Emprendimiento dentro de sus procesos formativos como lo señala la ley.1014 de 2006, el 60\% de las instituciones educativas en las que no se ha iniciado al proceso argumentan que la causa principal es la falta de información y capacitación sobre cómo llevar a cabo el proceso. En los colegios donde se inicio la implementación de la cátedra de formación para el emprendimiento solo se oferta desde los grados novenos en adelante. El 95\% de los docentes que orientan la cátedra en dichas instituciones no tienen formación profesional en áreas afines a las ciencias económicas, y solo un $10 \%$ ha recibido capacitación en temas relacionados con el emprendimiento y el desarrollo empresarial. En las instituciones públicas que están implementando la cátedra se presenta una dualidad a la hora de asignar carga académica para orientar la cátedra, pues algunos piensan que es una opción para descargarse de sus obligaciones en otras áreas, ya que solo tendrían que hablarles a sus estudiantes de ¿Qué es empresa?5, mientras otros

\footnotetext{
${ }^{3}$ Definiciones contenidas en el Modulo: Sandoval Casilimas. Carlos A. Investigación Cualitativa ICFES. Arfo Editores e Impresores Ltda. diciembre de 2002.

${ }_{4}^{4}$ Palabra utilizada de en esta investigación para referir las instituciones Educativas de Básica Primaria, secundaria y Media Técnica.

${ }^{5}$ La cátedra de formación para el emprendimiento en las escuelas debe tener conceptos y alcances más complejos que el solo termino "Empresa", argumento que no comprendían los docentes encuestados, pues se trata de desarrollar las características habilidades y valores para ser empresario y no solo de aprender en clase ¿que son las empresas?.
} 
docentes no desean saber nada al respecto, pues no les parece importante. Finalmente el 98\% de los docentes relaciona la cátedra de emprendimiento con "enseñar a los estudiantes a hacer empresa."

Con ese panorama inicio la investigación, que permitió evidenciar aspectos como la necesidad de hacer claridad sobre la metodología y herramientas requeridas para desarrollar procesos de formación para el emprendimiento. Después del análisis documental, el debate constructivo al interior del grupo de investigación y los aportes desde la experiencia profesional de los investigadores, se empezaron a construir las bases teóricas y metodologías de lo que debería ser la Formación para el emprendimiento en Básica Primaria Secundaria y Media Técnica.

El desarrollo del debate y análisis estructurado al interior del equipo de investigación, centralizo la discusión alrededor de tres ejes estratégicos cuyos componentes pueden afectar dichos proceso de formación, entre los que se desatacan:

\section{A. Los Factores internos y externos que favorecen o retrasan el proceso de institucionalización de la formación para el emprendimiento en básica primaria, secundaria y media técnica.}

\section{Externos}

Positivos: Las políticas planes, programas, proyectos, normas y leyes establecidas en Colombia para el fomento de la actividad emprendedora, la capacitación para el empleo y el desarrollo empresarial.

Negativos: La falta de herramientas pedagógicas y unanimidad de criterios sobre el fin de los procesos formativos en el tema de emprendimiento.

La escasez, de proceso investigativos, que orienten la labor formativa en el tema del emprendimiento y generen debates científicos sobre la pertinencia de los temas, a ser incluidos en los diseños curriculares.

El énfasis en el desarrollo de procesos de formación profesional integral para el empleo, descuidando la formación para la productividad desde el inicio de la educación escolar. Establecimiento del paradigma que de concepto e interpretación que iguala las "competencias laborales" $"$ ", con las "competencias para el emprendimiento", que

\footnotetext{
${ }^{6}$ El SENA, enfoca todos sus procesos formativos al desarrollo de acciones pedagógicas, tendientes al desarrollo de competencias laborales y profesionales que permiten el desarrollo de habilidades técnicas y sociales para desempeñarse eficientemente en el desarrollo de una labor, dependiendo de las exigencias que en materia laboral tengan las empresas, pero se enfoca en la "formación Profesional" de los individuos en edad productiva, por lo que de acuerdo con la investigación, tendría un papel preponderante en el proceso de formación para emprendedores en el desarrollo competencias, dimensión del hacer mas no en la en las del Ser y del saber previamente requerido para emprender.
} 
aunque son complementarias, difieren en su propósito; lo cual confunde a los educadores a la hora de decidirse por la utilización del modelo pedagógico.

\section{Internos}

Positivo: La especialización de las instituciones en un arte, oficio o labor en la media técnica a fin de promover la inserción de los graduados al mundo laboral., y facilitar sus procesos de formación posteriores mediante la modalidad de ciclos propedéuticos. La Reformulación de los P.E.I (Proyectos Educativos Institucionales), mediante la inserción de objetivos, tendientes a promover el la capacitación para el desarrollo de actividades productivas escolares.

Negativo: El Papel de los padres de familia, entidades gubernamentales, e instituciones de apoyo a los procesos complementarios de formación para el emprendimiento.

La falta de especialización o capacitación de los docentes de las instituciones educativas en emprendimiento, administración, desarrollo empresarial y temas afines.

\section{B. Aportes y alcances de la cualificación apropiada del capital humano, al desarrollo de una cultura del emprendimiento ${ }^{8}$ mediante la diferenciación entre características, valores y habilidades que deben desarrollar los emprendedores en sus procesos de formación.}

El equipo investigador concluye a este respecto que es importante hacer algunas aclaraciones de concepto que orienten el trabajo de los docentes por lo que hace nuevos aportes en cuanto a la definición de emprendedor, la clasificación de sus características, valores y habilidades.

El emprendedor. Debe ser un ser humano exitoso en todas las áreas de su vida, (personal, afectiva, espiritual, profesional, académica) de manera tal que pueda desarrollar sus capacidades creativas $^{9}$ e innovadoras ${ }^{10}$ potencializando sus características, valores y habilidades para la generación de bienes o servicios, en beneficio propio y de su entorno.

\footnotetext{
${ }^{7}$ Los procesos de formación para el emprendimiento desde la edad escolar suponen el desarrollo de competencias no laborales, exclusivamente si personales que le permitan al estudiante el desarrollo de características habilidades y valores para ser empresario y no solo de aprender en clase ¿que son las empresas? O aprender cómo ser buen vendedor o como llevar bien la contabilidad o producir un servicio especifico.

8 CULTURA DEL EMPRENDIMIENTO: Conjunto de valores, creencias, ideologías, hábitos, costumbres, normas y patrones de comportamiento colectivos, tendientes a desarrollar procesos efectivos que permitan la creación de empresas, generación de empleo y desarrollo socioeconómico en una sociedad. 9 CREATIVIDAD: Entendida como la capacidad de desarrollar ideas de negocios, que mejoren proceso de producción o comercialización de productos servicios ofertados en el mercado.

10 INNOVACION: Entendida como la capacidad de desarrollar ideas de negocios, producto de investigaciones, en las que se evidencie el desarrollo tecnológico y puedan incluso ser patentados.
} 


\section{Características:}

a) LIDERAZGO, Entendido como la forma como influimos sobre los demás por medio de la autoridad y el poder (legítimo, de recompensa, coercitivo, experto, de las relaciones), en busca de objetivos concretos de beneficio colectivo.

b) TRABAJO EN EQUIPO: Capacidad de, Contribuir al logro de metas y objetivos dentro de un grupo, cuyos miembros tienen Habilidades que se complementan, y son interdependientes lo cual garantiza el éxito.(SINERGIA ${ }^{11}$ ).

c) CONOCIMIENTO: Capacidad de apropiación de conocimiento e información de su interés indispensable para desarrollar ventajas competitivas, propias de la labor empresarial.

d) GESTION: Acción permanente tendiente a la búsqueda de los medios que le permitan alcanzar sus fines u objetivos.

e) MOTIVACION: Estado o condición que le permite a un individuo, identificar y priorizar sus necesidades para encauzar sus esfuerzos al logro de sus objetivos.

\section{Valores:}

a) RESPONSABILIDAD ETICA: entendida como la capacidad de realizar actos humanos trascendentes con voluntad y libertad absoluta, de la persona, asumiendo sus consecuencias sean estas positivas o negativas

b) B ESPIRITUALIDAD: es el ejercicio natural que todo ser con conciencia hace del Vigor natural o fortaleza que provee el reconocer la existencia de DIOS, que alienta a obrar con; ánimo, valor, aliento, brío, esfuerzo, vivacidad y/o ingenio.

c) DISCIPLINA: entendida como la capacidad de actuar ordenada y perseverantemente para conseguir beneficio individual o colectivo.

d) AUTOESTIMA12: Puede definirse como el sentimiento de aceptación y aprecio hacia uno mismo, que va unido al sentimiento de competencia y valía personal.

\footnotetext{
${ }^{11} \mathrm{El}$ término consiste en que se consiguen ventajas en el trabajo asociado. Es el efecto adicional que dos organismos obtienen por trabajar de común acuerdo, la sinergia es la suma de energías individuales que se multiplica progresivamente, reflejándose sobre la totalidad del grupo. La valoración de las diferencias (mentales, emocionales, psicológicas) es la esencia de la sinergia. Y la clave para valorar esas diferencias consiste en comprender que todas las personas ven el mundo no como es, sino como son ellas mismas. Puede verse en http://www.sinergiacolombia.org/003porque.htm.

12 El concepto que tenemos de nosotros mismos no es algo heredado, sino aprendido de nuestro alrededor, mediante la valoración que hacemos de nuestro comportamiento y de la asimilación e interiorización de la opinión de los demás respecto a nosotros. La importancia de la autoestima radica en que nos impulsa a actuar, a seguir adelante y nos motiva para perseguir nuestros objetivos.
} 


\section{Habilidades:}

a) COMUNICACIÓN: Capacidad para acceder, seleccionar, manejar e intercambiar información que facilite la toma de decisiones y permita establecer relaciones comerciales confiables, con clientes o proveedores en el ejercicio de su roll como empresario.

b) B) NEGOCIACION DE CONFLICTOS Negociar consiste en discutir un asunto para llegar a un acuerdo que suponga una satisfacción para las partes involucradas en el mismo. Esta alternativa da lugar a vencedores ni vencidos, ambas partes ganan y pierden algo, por lo que el emprendedor debe saber, las razones costo beneficio de perder o ganar en una negociación, para la toma de decisiones adecuadas.

c) MANEJO DE NUEVAS TECNOLOGIAS: entendida como la habilidad para comunicarse con el mundo haciendo uso de todas las tecnologías informáticas disponibles.

d) NEGOCIACION EMPRESARIAL ${ }^{13}$ : Entendida como la capacidad de aumentar la eficacia del comprador empresarial al definir sus deberes, responsabilidades, habilidades y acciones específicas en el proceso de negociación con el vendedor.

e) HABILIDADES GERENCIALES14: son un conjunto de competencias y conocimientos que una persona posee para realizar las actividades de administración de los recursos disponibles.

\section{Pertinencia y Estructura del Diseño curricular de formación para el Emprendimiento.}

La formación para el emprendimiento no debe relacionarse solo con el desarrollo de Competencias laborales ya que estas son solo la última etapa en los procesos de formación para el emprendimiento; por lo que para hacer más eficaces dichos procesos debe estructurarse un diseño curricular apropiado regido por los principios de integralidad, coherencia, pertinencia, secuencialidad y permanencia, que promueva el

13 ALDANA HERRERA, FRANCISCO EL ARTE DE LA NEGOCIACIÓN EMPRESARIAL. MANUAL DEL COMPRADOR EFICAZ ISBN: 8466573259 N PÁG: 308 mayo de 2007 .El texto se basa en los dos aspectos de la negociación, la compra y la venta; su enfoque se centra en las negociaciones que se realizan en el área de compras de una empresa, y su objetivo es poner al alcance del empresario, comprador o vendedor, los conocimientos teóricos y técnicos que le permitan lograr la eficacia en todas sus negociaciones.

14 Dependiendo del nivel gerencial, se desarrollan otras habilidades, clasificadas como: a) Habilidades técnicas: Involucra el conocimiento y experticia en determinados procesos, técnicas o herramientas propias del cargo o área especifica que ocupa. b) Habilidades Humanas: Se refiere a la habilidad de interactuar efectivamente con las personas. Un gerente interactúa y coopera principalmente con los empleados a su cargo; muchos también tienen que tratar con clientes, proveedores, aliados, etc.) Habilidades Conceptuales: Se trata de la formulación de ideas, entender relaciones abstractas, desarrollar nuevos conceptos, resolver problemas en forma creativa, etc. 
desarrollo de competencias emprendedoras básicas y transversales en las dimensiones del ser, del saber, y del hacer.

El producto final de la reflexión, el debate, y la consulta, fue el desarrollo de la propuesta metodológica denominada "Ruta de Formación para el Emprendimiento" la cual no pretende reemplazar textos y demás herramientas existentes para la orientación de las temáticas relacionada con administración o generación de empresa y emprendimiento; por el contrario ordena de manera progresiva el desarrollo de los contenidos ${ }^{15}$ para facilitar la formación en el emprendimiento de los Colegios. La ruta está compuesta de 7 matrices donde se describe: el nombre del modulo para cada grado, las dimensiones ${ }^{16}$ que se abordan; las unidades temáticas en desarrollo y los contenidos para cada una de ellas, de manera que el docente pueda elaborar la planeación de actividades y evaluación periódica de las mismas.

\section{CONCLUSIONES}

Existen factores internos y externos que favorecen o retrasan el proceso de institucionalización de la formación para el emprendimiento en básica primaria, secundaria y media técnica, por lo que se deben prever las intervenciones para minimizar sus efectos.

Entre los aportes a la cualificación apropiada del capital humano, desde la reflexión del equipo investigador se desataca la conceptualización sobre el emprendedor, sus características, valores y habilidades que deben ser tenidos en cuenta al desarrollar y evaluar procesos de formación para emprendedores en los Colegios.

La estandarización de procesos metodológicos que orienten la formación para el Emprendimiento facilita la evaluación de resultados que evidencien el impacto de los proceso de formación para el emprendimiento en la generación de una verdadera cultura emprendedora; pero además se convierte en una herramienta de trabajo y debate que facilita nuevas construcciones metodológica similares.

\footnotetext{
15 Ver Tablas anexas con la ruta de formación desde grado cero hasta undécimo.

${ }^{16}$ Competencias dimensión del ser: Características del emprendedor: Conocimiento de sí mismo (autoestima). de sus capacidades para trabajar en equipo, liderazgo, Motivación, comunicación y los valores. Competencia dimensión del saber: Conocimiento de su Entorno. Conocimiento de conceptos y principios administrativos. Conocimiento de las fuentes de financiación y formación técnica o en un arte u oficio. Competencias dimensión del hacer: Se refiere a la capacidad de ser creativo e innovador, proponiendo ideas de negocio o actividades productivas que contribuyan a generar ingresos, bienestar para sí mismo y para la sociedad a la que pertenece, generando impacto positivo en su entorno y participando activamente en el desarrollo socioeconómico local y nacional.
} 
Tablas anexas (Ruta de Formación para el Emprendimiento, Contenidos).

$\boldsymbol{T A B L A}$ 1. Modulo básica primaria, grado 0 a 5: "¿que quiero ser cuando grande? Y ¿que necesito para lograrlo?

\begin{tabular}{|c|c|}
\hline $\begin{array}{l}\text { UNIDADES } \\
\text { TEMATICAS }\end{array}$ & CONTENIDOS \\
\hline $\begin{array}{l}\text { GRADO } 0 \\
\text { LAS COSAS QUE } \\
\text { ME AGRADAN, Y } \\
\text { A LAS QUE LES } \\
\text { TEMO. }\end{array}$ & $\begin{array}{l}\text { - ¿Quién soy? } \\
\text { - ¿Cómo Vivo? } \\
\text { - ¿Qué hacen las personas que me rodean? } \\
\text { - } \quad \text { Las cosas que me enojan y las cosas que me agradan. }\end{array}$ \\
\hline $\begin{array}{l}\text { GRADO } 1 . \\
\text { LAS COSAS QUE } \\
\text { ME GUSTARIA } \\
\text { HACER CUANDO } \\
\text { CREZCA. }\end{array}$ & $\begin{array}{l}\text { - Los oficios, las profesiones, las artes } \\
\text { - } \quad \text { Las cosas que me gustan y me disgustan de ser un } \\
\text { perdedor o un ganador. }\end{array}$ \\
\hline $\begin{array}{l}\text { GRADO } 2 \\
\text { COMO GANARE } \\
\text { DINERO } \\
\text { CUANDO SEA } \\
\text { GRANDE }\end{array}$ & $\begin{array}{l}\text { - ¿Qué me gustaría tener cuando sea grande?, y ¿Cómo } \\
\text { ganare dinero para conseguirlo? } \\
\text { - ¿Conozco el valor del dinero? }\end{array}$ \\
\hline $\begin{array}{l}\text { GRADO } 3 \\
\text { COMO GANAN } \\
\text { DINERO PAPA Y } \\
\text { MAMA }\end{array}$ & $\begin{array}{l}\text { - En que se ocupan las personas para ganar dinero? } \\
\text { - ¿as cosas que consumimos, ¿Quién las produce? } \\
\text { - ¿Cómo lo hacen? } \\
\text { mis padres? }\end{array}$ \\
\hline $\begin{array}{l}\text { GRADO } 4 . \\
\text { HOMBRES Y } \\
\text { MUJERES DE } \\
\text { ÉXITO EN LA } \\
\text { HISTORIA. }\end{array}$ & $\begin{array}{l}\text { - Como surgieron los grandes inventos. } \\
\text { - } \quad \text { Historias de vida exitosas. Características y rasgos de } \\
\text { - } \text { personalidad que los hicieron grandes } \\
\text { - } \text { quien admiro y ¿Por qué? }\end{array}$ \\
\hline $\begin{array}{l}\text { GRADO } 5 \\
\text { NIÑOS } \\
\text { EMPRENDEDOR } \\
\text { ES HACIENDO } \\
\text { NEGOCIOS. }\end{array}$ & $\begin{array}{l}\text { - ¿Por qué necesito de las personas para que me ayuden } \\
\text { a ganar dinero? } \\
\text { - } \quad \text { Negociando soy un ganador. } \\
\text { - } \quad \text { Ganando mi propio dinero. } \\
\text { Ahorrando, una forma de alcanzar mis metas. }\end{array}$ \\
\hline
\end{tabular}


TABLA 2. Modulo sexto grado: "el emprendedor, el mundo y el dinero"

\begin{tabular}{|c|c|}
\hline UNIDADES TEMATICAS & CONTENIDOS \\
\hline $\begin{array}{l}\text { UNIDAD } 1 . \\
\text { CONCEPTUALIZACION }\end{array}$ & 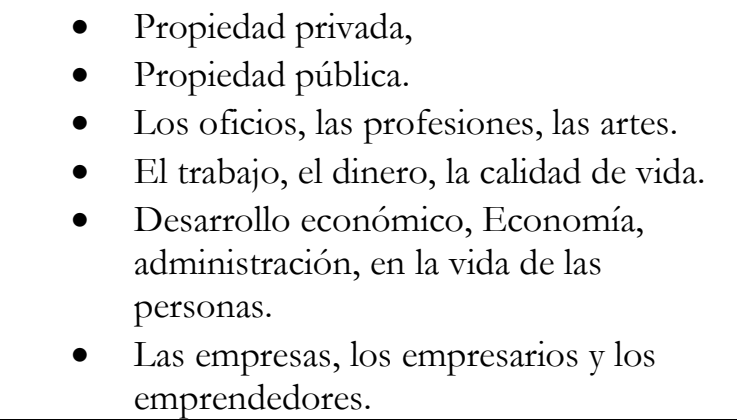 \\
\hline $\begin{array}{l}\text { UNIDAD } 2 \text { ¿DONDE } \\
\text { TRABAJAN LAS PERSONAS? }\end{array}$ & $\begin{array}{l}\text { - ¿En que se ocupan las personas para } \\
\text { ganar dinero? Producción de bienes, } \\
\text { producción de servicios. } \\
\text { Diferencia entre los empleos } \\
\text { permanentes, temporales e } \\
\text { independientes, formas de contratación, } \\
\text { pago y beneficios. } \\
\text { ¿Quiénes pueden, dar empleo en el país? } \\
\text { Las instituciones públicas y el empleo. } \\
\text { Las empresas y el empleo en el país. } \\
\text { Estudio de casos sobre la situación del } \\
\text { desempleo en el país, el departamento y } \\
\text { el municipio. } \\
\text { La alternativa de emprender para ganar } \\
\text { dinero y generar empleo. }\end{array}$ \\
\hline $\begin{array}{l}\text { UNIDAD } 3 . \\
\text { LA MOTIVACION PARA } \\
\text { HACER DINERO Y } \\
\text { EMPRENDER }\end{array}$ & $\begin{array}{l}\text { La satisfacción de necesidades y la } \\
\text { motivación para buscar trabajo, } \\
\text { emprender y ganar dinero. } \\
\text { - La motivación, mis intereses, mis } \\
\text { necesidades y mis logros. } \\
\text { - Proyecto de vida escolar, personal, } \\
\text { profesional. } \\
\text { Elaboración del Plan estratégico } \\
\text { personal emprendedor }\end{array}$ \\
\hline
\end{tabular}




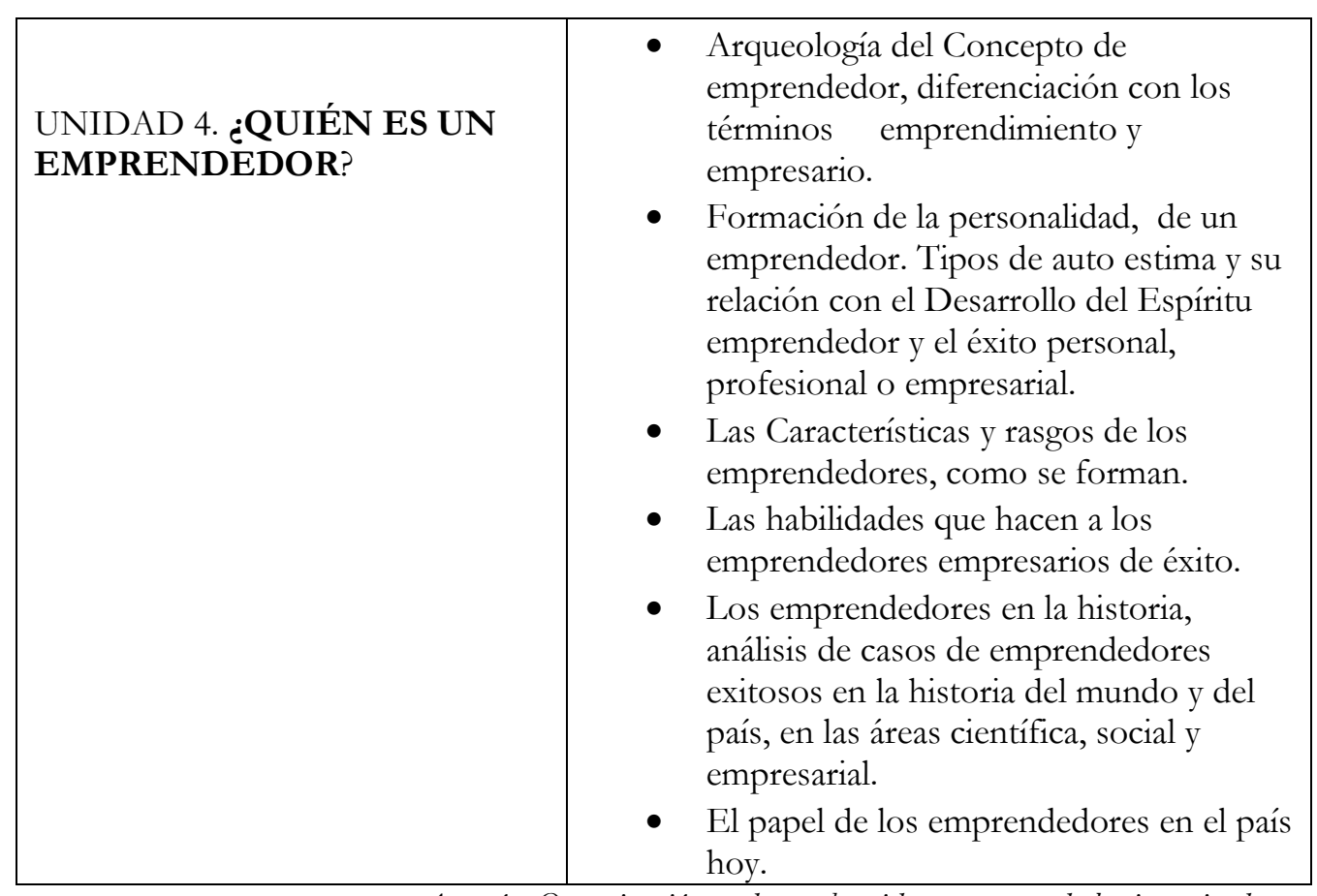

Autoría, Organización y datos obtenidos por parte de los investigadores

TABLA 3. Modulo séptimo grado: "escenarios de construcción y manejo de las relaciones interpersonales del emprendedor".

\begin{tabular}{|l|ll|}
\hline \multicolumn{1}{|c|}{ UNIDADES TEMATICAS } & \multicolumn{2}{c|}{ CONTENIDOS } \\
\hline UNIDAD 1. & $\bullet$ & Concepto y estilos de liderazgo, \\
EL EJERCICIO DEL & $\bullet$ & Características de los líderes \\
LIDERAZGO Y LAS & & emprendedores y relación con la \\
RELACIONES DE PODER. & & toma de decisiones individuales. \\
& $\bullet$ & Los líderes y el ejercicio del poder. \\
& $\bullet$ & Vínculos entre los términos \\
& & empresa, administración, \\
& emprendedores y empresarios. \\
\hline
\end{tabular}




\begin{tabular}{|c|c|}
\hline $\begin{array}{l}\text { UNIDAD } 2 \\
\text { EL TRABAJO DE GRUPO, DE } \\
\text { EQUIPO Y SU RELACION CON } \\
\text { LA PRODUCTIVIDAD.. }\end{array}$ & $\begin{array}{l}\text { - Los grupos formales e informales } \\
\text { eficaces. } \\
\text { - Tipos de equipos de trabajo. } \\
\text { - El desarrollo de las habilidades de } \\
\text { equipo, para la toma de decisiones } \\
\text { colectivas. } \\
\text { - Acciones y actitudes relacionadas } \\
\text { con el rol de las personas dentro de } \\
\text { los equipos. } \\
\text { - Ventajas, desventajas y situaciones } \\
\text { de aplicación del trabajo en equipo } \\
\text { en las empresas y organizaciones. }\end{array}$ \\
\hline $\begin{array}{l}\text { UNIDAD } 3 . \\
\text { LA NEGOCIACION Y MANEJO } \\
\text { DE CONFLICTOS } \\
\text { INDIVIDUALES Y COLECTIVOS. }\end{array}$ & $\begin{array}{l}\text { - Los conflictos, individuales y } \\
\text { colectivos. } \\
\text { - El papel de los actores involucrados } \\
\text { e el desarrollo de un conflicto. } \\
\text { - Técnicas de Negociación de } \\
\text { Conflictos de intereses en el aula y } \\
\text { en la vida cotidiana. }\end{array}$ \\
\hline $\begin{array}{l}\text { UNIDAD } 4 . \\
\text { EL EMPRENDEDOR ESCOLAR } \\
\text { COMO LIDER, Y NEGOCIADOR. }\end{array}$ & $\begin{array}{l}\text { - Análisis de las relaciones de de } \\
\text { Poder dentro del aula, situaciones de } \\
\text { conflicto y manejo de los mismos. } \\
\text { - Identificando potenciales, lideres } \\
\text { Emprendedores. } \\
\text { - La participación como instrumento } \\
\text { de poder colectivo. }\end{array}$ \\
\hline
\end{tabular}

Autoría, Organización y de datos obtenidos por parte de los investigadores 
TABLA 4. MODULO OCTAVO GRADO: "INSTITUCIONALIDAD Y LAS ORGANIZACIONES EMPRESARIALES”

\begin{tabular}{|c|c|}
\hline UINIDADES TEMATICAS & CONTENIDOS \\
\hline $\begin{array}{l}\text { UNIDAD 1. CONCEPTUALIZACION } \\
\text { SOBRE CREACION Y MANEJO DE, } \\
\text { ORGANIZACIONES, EMPRESAS E } \\
\text { INSTITUCIONES. }\end{array}$ & 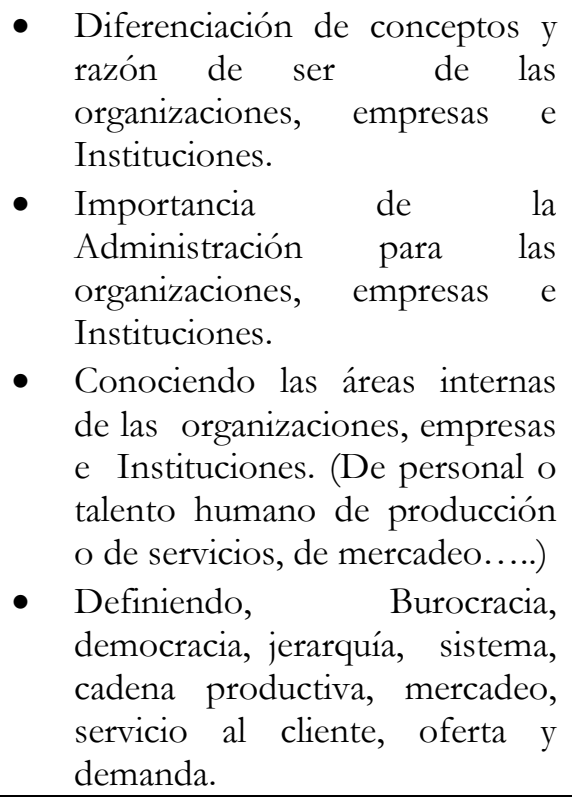 \\
\hline $\begin{array}{l}\text { UNIDAD } 2 \text { IMPORTANCIA DE LA } \\
\text { CLASIFICACION, } \\
\text { DIFERENCIACION Y } \\
\text { CARACTERIZACION DE LAS } \\
\text { ORGANIZACIONES, PARA EL } \\
\text { EMPRENDEDOR. }\end{array}$ & $\begin{array}{l}\text { - Las Instituciones del estado. } \\
\text { - Las empresas públicas, en el } \\
\text { país, la región y el municipio. } \\
\text { Las empresas de Economía } \\
\text { mixta país, la región y el } \\
\text { municipio. } \\
\text { - Las empresas privadas } \\
\text { dedicadas a la producción de } \\
\text { bienes y servicios en el país. } \\
\text { Tipología de las empresas } \\
\text { privadas. (Sociedades, } \\
\text { corporaciones, consorcios, } \\
\text { grupos empresariales, } \\
\text { Cooperativas....) } \\
\text { Las Organizaciones sociales, y } \\
\text { comunitarias. (ONG, } \\
\text { Fundaciones, Asociaciones, } \\
\text { Juntas de acción comunal...) }\end{array}$ \\
\hline
\end{tabular}


UNIDAD 3. CONSTRUCCION DEL MAPA EMPRESARIAL Y LABORAL COLOMBIANO.

Autoría, Organización y de datos obtenidos por parte de los investigadores

- Los sectores económicos, más representativos, en cuanto a producción y generaron de empleo.

- Que son y que muestran los indicadores económicos, PIB, inflación, IPC, IVA,.....

- Las políticas, planes de desarrollo, programas y proyectos, destinados a la generación de empleo y el desarrollo empresarial en el país.

- El país en el que vivo, se mueve al ritmo del mundo.

TABLA 5. Modulo noveno grado: "el entorno empresarial y el desarrollo socioeconómico, regional y nacional y mundial"

\begin{tabular}{|c|c|}
\hline UINIDADES TEMATICAS & CONTENIDOS \\
\hline $\begin{array}{l}\text { UNIDAD 1. LA GLOBALIZACION } \\
\text { ECONOMICA. }\end{array}$ & $\begin{array}{l}\text { - } \text { El comercio internacional de productos } \\
\text { y servicios importaciones, } \\
\text { exportaciones. Tratados, convenios y } \\
\text { pactos y asociaciones entre países. } \\
\text { - Concepto de globalización, } \\
\text { - Países desarrollados, países del tercer } \\
\text { mundo, ¿Qué compran y que vende? } \\
\text { - ¿Por qué los emprendedores deben } \\
\text { saber lo que ocurre en el mundo? } \\
\text { - Casos de empresas existas en el mundo } \\
\text { que aplican el concepto de } \\
\text { Globalización }\end{array}$ \\
\hline $\begin{array}{l}\text { UNIDAD 2. LOS TIPOS DE } \\
\text { ENTORNO QUE AFECTAN EL } \\
\text { DESARROLLO EMPRESARIAL, } \\
\text { LA LABOR DE LOS } \\
\text { EMPRESARIOS Y } \\
\text { EMPRENDEDORES. }\end{array}$ & $\begin{array}{ll}\text { - } & \text { Entorno tecnológico. } \\
\text { - } & \text { Entorno, educativo. } \\
\text { - } & \text { Entorno Normativo y legislativo, } \\
\text { - } & \text { Entorno Sociocultural. } \\
\text { - } & \text { Entorno político y Gubernamental Económico y financiero. } \\
\text { - } & \text { Entorno Ambiental. } \\
\text { - } & \text { Entorno Geográfico. }\end{array}$ \\
\hline $\begin{array}{l}\text { UNIDAD 3. LAS AGENDAS DE } \\
\text { COMPETITIVIDAD REGIONAL } \\
\text { Y NACIONAL. }\end{array}$ & $\begin{array}{llll}\text { - } & \text { ¿Qué son las agendas } & \text { de } \\
& \text { Competitividad nacional y regional? } & \\
\text { - } & \text { Quien las elabora las agendas de }\end{array}$ \\
\hline
\end{tabular}




\begin{tabular}{|c|c|}
\hline & $\begin{array}{l}\text { competitividad y con qué propósito } \\
\text { ¿Qué relación existe entre el desarrollo } \\
\text { de las agendas de competitividad, y el } \\
\text { desarrollo de planes y programas de } \\
\text { gobierno encaminados a fomentar la } \\
\text { creación de empresas. } \\
\text { Como participar en la elaboración de las } \\
\text { A.C. }\end{array}$ \\
\hline $\begin{array}{l}\text { UNIDAD } 4 . \\
¿ \text { ¿ONDE ENCONTRAR LAS } \\
\text { OPORTUNIDADES DE } \\
\text { NEGOCIOS? }\end{array}$ & $\begin{array}{l}\text { - Elaboración de análisis de } \\
\text { oportunidades de negocio, en el mundo, } \\
\text { el país y la región. } \\
\text { - } \begin{array}{l}\text { Quienes invierten en los nuevos } \\
\text { negocios. } \\
\text { - Como se financian las empresas } \\
\text { existentes. }\end{array}\end{array}$ \\
\hline
\end{tabular}

Autoria, Organización y datos obtenidos porparte de los investigadores

TABLA 6. Modulo decimo grado: "el mundo de las empresas y los empresarios"

\begin{tabular}{|c|c|}
\hline UINIDADES TEMATICAS & CONTENIDOS \\
\hline $\begin{array}{l}\text { UNIDAD } 1 . \\
\text { INNOVACION, } \\
\text { CREATIVIDAD E IDEAS DE } \\
\text { NEGOCIOS. }\end{array}$ & $\begin{array}{l}\text { - Innovar una forma de hacer empresa } \\
\text { investigando. Análisis de Casos de } \\
\text { empresas, que emprendieron ideas de } \\
\text { negocio innovadoras } \\
\text { - La creatividad en la generaron de ideas } \\
\text { de negocios, una forma de transformar } \\
\text { el mundo que nos rodea, mediante la } \\
\text { satisfacción de las necesidades, gustos e } \\
\text { intereses de otros. } \\
\text { - Generando ideas de negocios, creativas } \\
\text { o innovadoras, en un mundo globalizad } \\
\text { Proyecto productivo escolar, como } \\
\text { idea de negocio. }\end{array}$ \\
\hline $\begin{array}{l}\text { UNIDAD } 2 \\
\text { CASOS DE } \\
\text { EMPRENDIMIENTO } \\
\text { BASADOS EN PROCESOS } \\
\text { CREATIVOS O } \\
\text { INNOVADORES. }\end{array}$ & $\begin{array}{l}\text { - Estudio de casos de emprendimiento a } \\
\text { nivel MUNDIAL donde se involucran } \\
\text { procesos creativos o innovadores, } \\
\text { análisis de las historia de vida e } \\
\text { historias empresariales. } \\
\text { - Estudio de casos de emprendimiento a } \\
\text { nivel NACONAL donde se involucran } \\
\text { procesos creativos o innovadores, }\end{array}$ \\
\hline
\end{tabular}




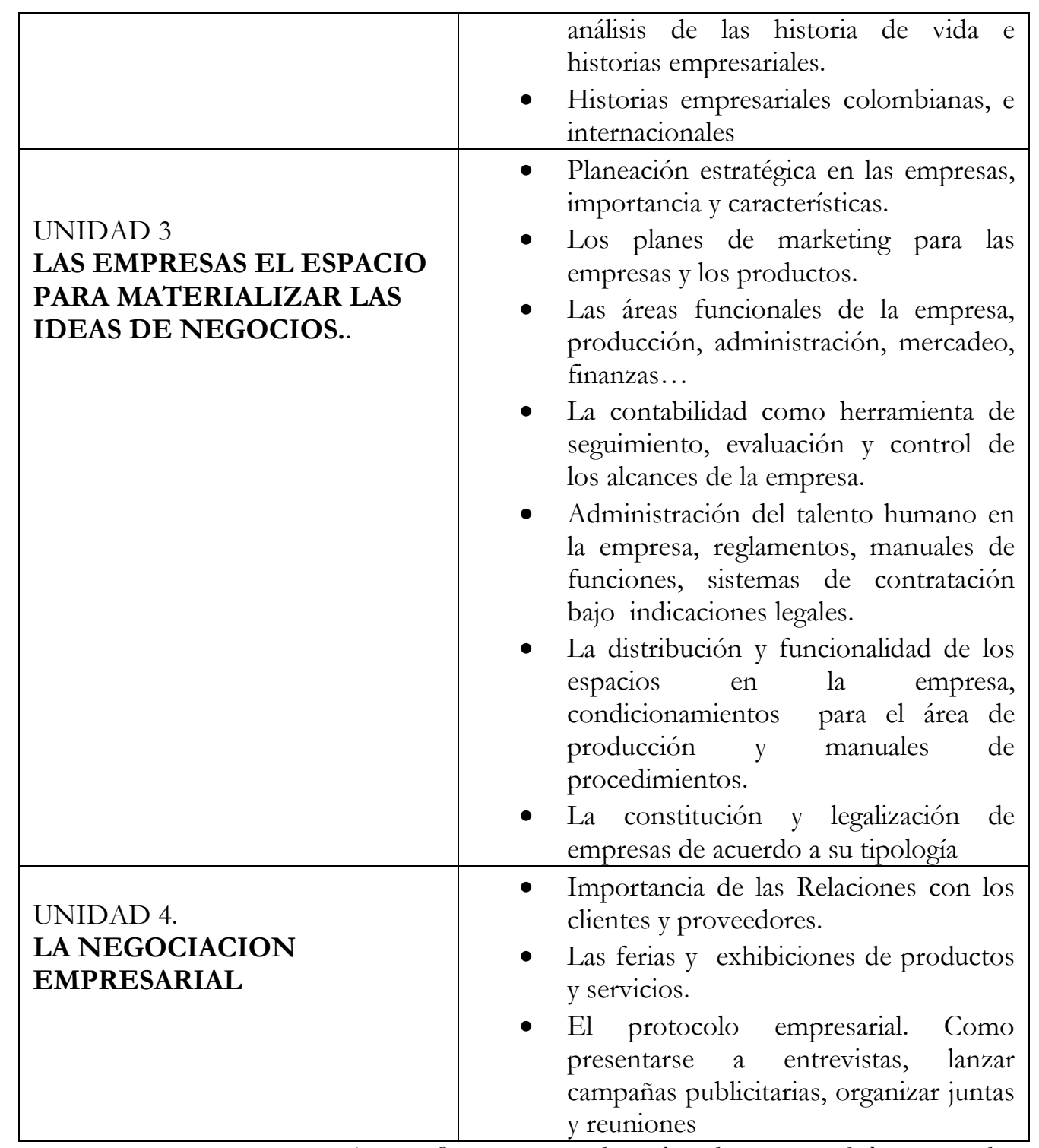

Autoria, Organización y datos obtenidos por parte de los investigadores 
TABLA 7. Modulo undécimo grado: “emprendiendo mi propio negocio."

\begin{tabular}{|c|c|}
\hline UINIDADES TEMATICAS & CONTENIDOS \\
\hline $\begin{array}{l}\text { UNIDAD } 1 . \\
\text { PROYECTO PRODUCTIVO } \\
\text { ESCOLAR }\end{array}$ & $\begin{array}{l}\text { - } \\
\text { El merado de los productos y servicios en el } \\
\text { - } \\
\text { - } \\
\text { Elección de una actividad productiva. } \\
\text { - } \\
\text { fines. } \\
\text { Actividad productiva escolar }\end{array}$ \\
\hline $\begin{array}{l}\text { UNIDAD } 2 \\
\text { IMAGEN CORPORATIVA Y } \\
\text { COMUNICACIÓN } \\
\text { EMPRESARIAL }\end{array}$ & $\begin{array}{l}\text { - Parámetros para el diseño de imagen } \\
\text { corporativa. Elaboración de portafolios } \\
\text { empresariales. } \\
\text { - Importancia de la sistematización de los } \\
\text { procesos de comunicación empresarial, para } \\
\text { mejorar su eficiencia, y facilitar los proceso de } \\
\text { certificación. } \\
\text { Canales de Comunicación formal e informal, y } \\
\text { desarrollo de tácticas para superar los } \\
\text { obstáculos de comunicación. } \\
\text { La Comunicación Externa, formas lecturas, } \\
\text { interpretación, e importancia en la toma de } \\
\text { decisiones empresariales. } \\
\text { Diseño y ejecución de de Campañas } \\
\text { publicitarias para productos y organizaciones } \\
\text { empresariales en distintos medios de } \\
\text { comunicación. }\end{array}$ \\
\hline $\begin{array}{l}\text { UNIDAD } 3 . \\
\text { ELABORACION DE } \\
\text { PLANES DE NEGOCIOS }\end{array}$ & 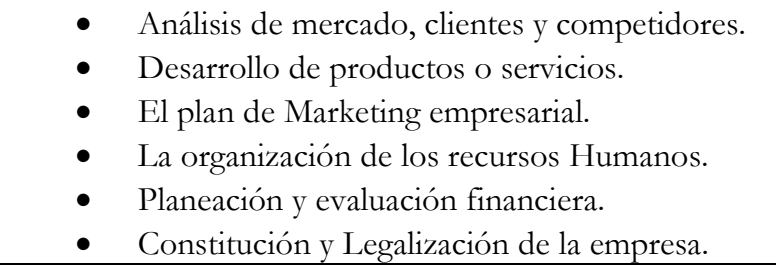 \\
\hline $\begin{array}{l}\text { UNIDAD } 4 . \\
\text { FUENTES DE } \\
\text { FINANCIACION DE } \\
\text { PROYECTOS }\end{array}$ & $\begin{array}{l}\text {-La Financiación de proyectos mediante planes } \\
\text { y programas nacionales de Desarrollo } \\
\text { Empresarial. Instituciones, requerimientos } \\
\text { - La financiación de Proyectos producto de la } \\
\text { Responsabilidad social empresarial. } \\
\text { - Análisis del Manual de Fuentes de Financiación } \\
\text { Nacional, Departamental y local. } \\
\text { - La financiación de Proyectos mediante } \\
\text { Cooperación Internacional metodología y } \\
\text { acceso a las fuentes. }\end{array}$ \\
\hline
\end{tabular}

Autoría, Organización y de datos obtenidos por parte de los investigadores 


\section{REFERENCIAS}

- BARBOSA, Cano Erasmo, Calidad Total para juntas y Reuniones. Ed. McGraw-Hill Colombia. 1996.

- Biblioteca práctica de Negocios, Tomos I. II. III. IV, V, VII, VIII. Ed. McGrawHill México 1996.

- BONO, Edward de. El Pensamiento Creativo. El poder del pensamiento lateral para la creación de nuevas ideas. Ediciones Paidos. Barcelona, España. 1994.

- BRAVO SALINAS, Néstor. Pedagogía Problemática. Acerca de los nuevos paradigmas en educación. Convenio Andrés Bello. Santa Fe de Bogotá, 1997.

- CATAlanO, A. (coord.) Enseñar y evaluar en formación por competencias laborales: orientaciones conceptuales y metodológicas. Primera Edición. Buenos Aires: BID/FOMIN; CINTERFOR/OIT, 2006.

- DE IBALRROBA, María. Formación Escolar para el Trabajo. Ed. Cinterfor, Montevideo, Agosto de 2006.

- DE ZUBIRÍA, Miguel y DE ZUBIRÍA, Julián. Biografía del Pensamiento. Estrategias para el desarrollo de la inteligencia. Ediciones Antropos Ltda. Santafé de Bogotá, 1992.

- DUBRIN, Andrew J, Fundamentos de Administración. Thompson Editores .Colombia 2000.

- FIGUERAS, F., Marketing Estratégico integra. Primera Ed., Thompson Editores 2002, 220 pág.

- GAllegO-BADIllo, Rómulo. Discurso sobre el Constructivismo. Rojas Eberhard Editores. Santafé de Bogotá, 1993

- GIDO, Jack /CLEMENTS James. Administración Exitosa de proyectos. Segunda Ed., Thompson Editores2003 .526 Pág.

- GOMEZ, López Ernesto Javier. Análisis e Interpretación de Estados Financieros. Segunda Ed., Thompson Editores 2000 .107 Pág.

- GONZÁLEZ, Cuberes María Teresa Manual para diseñar estructuras curriculares y módulos de formación para el desarrollo de competencias en la formación profesional integral. El taller de los Talleres. Buenos Aires, Indugraf, 1987.

- GUITMAN, Larry j., MCDANIEL, Carl. El Futuro de Los Negocios, Tercera Ed., Thompson Editores 2000 .704 Pág.

- HAGER, Paul y BECKETT, David. Bases Filosóficas del Concepto Integrado de Competencia. En el libro "Competencia Laboral y Educación Basada en Normas de Competencia”; compilado por Antonio Argüelles, Noriega Editores, México 1996.

- HARRINGTON, H. J. Mejoramiento de procesos en La Empresa. Ed. McGRAWHILL Colombia. 1996 
“...RUTA DE FORMACIÓN PARA EL EMPRENDIMIENTO EN

EDUCACIÓN BÁSICA PRIMARIA, SECUNDARIA

Y MEDIA TÉCNICA...” EN COLOMBIA.

- HELlRIEGEL, Don; JACKSON, Susan; SLOCUM, John. Administración un Enfoque basado en Competencias. Novena Ed. Thompson Editores 2002 . Pag.561.

- HERNÁNDEZ, Fernando y SANCHO, Juana María. Para enseñar no basta con saber la asignatura. Paidós, Papeles de Pedagogía.1996

- ICONTEC. Instituto Colombiano de Normas Técnicas y Certificación. Norma Técnica Colombiana. NTC-ISO 9000:2000

- JURADO, Rojas Yolanda, Técnicas de Investigación Documental. Primera Ed., Thompson Editores 2002 .236 Pág.

- LUSSIER, Robert N. / ACHUA, Christopher F. Liderazgo, Teoría aplicación y Desarrollo de habilidades. Primera Ed., Thompson Editores 2001. Pág. 478.

- MERTENS, Leonard. Competencia Laboral: Sistemas, Surgimiento y Modelos. OIT. Cinterfor. Montevideo. 1996

- MIRANDA, Juan José. El Desafió de La Gerencia de proyectos, Segunda ed., Guadalupe Ltda. Bogotá D.C. Colombia.2006.

- MIRANDA, Juan José. Gestión de proyectos, Quinta ed., Guadalupe Ltda. Bogotá D.C. Colombia. 2005.

- MODUlOS DiAlogO DE GESTIONES, I, II, III, IV, V, VII, VIII. Centro de Acción Microempresarial, Programa Destapa Futuro. Colombia 2006.

- MORENO. Avendaño José del Carmen. Economía y Solidaridad, UNAD, Bogotá 2002.

- PATIÑO G., C.A. Modelos de calidad en la formación profesional y en la educación. Análisis y complementariedad. Ed.CINTERFOR/OIT, Montevideo: 2006.

- REZA, Trosino Jesús Carlos, El Empresario Hábil 2. Primera Ed., Thompson Editores 1997. Pág. 103.

- SENA. Dirección General. Formación Profesional Basada en Competencias: Estado actual y perspectivas. Hacia un modelo institucional. Santafé de Bogotá, 1996.

- VARGAS, F. Competencias clave y aprendizaje permanente. Ed. Cinterfor, Montevideo: 2004.

- Ley 344 de 1996, por la cual se dictan normas tendientes a la racionalización del gasto público, en su Artículo 16 obliga al SENA a destinar sus ingresos al desarrollo de programas de competitividad y de desarrollo productivo. Ley 550 de 1999, o ley de Intervención Económica y Reactivación Empresarial. Ley 29 de 1990, llamada Ley de Ciencia y Tecnología, es el marco que regula las disposiciones para el fomento del desarrollo tecnológico, y la promoción de empresas basadas en innovación y desarrollo tecnológico. Ley 590 del 10 de julio de 2000, conocida como Ley MIPYME. Fue creada principalmente con el objeto de "Inducir el establecimiento de mejores condiciones del entorno institucional para la creación y operación de micro, pequeñas y medianas empresas"; Reformada por la Ley 905 de agosto de 2004, 
Creación del FOMYPIME. Decreto 934 de 2003. Fondo Emprender. Tiene como objeto exclusivo financiar iniciativas empresariales que provengan y sean desarrolladas por aprendices o asociaciones entre aprendices, practicantes universitarios o profesionales, cuya formación se esté desarrollando o se haya desarrollado en las Instituciones reconocidas por el Estado, de conformidad con las Leyes 30 de 1992 y 115 de 1994. La ley 1014 de 2006, o ley de Fomento a la Cultura del Emprendimiento, tiene como objeto "Promover el espiritu emprendedor en todos los estamentos educativos del país.

- KANTIS, Hugo. (PhD) Universidad Nacional de General Sarmiento, quien publico el libro Titulado "Desarrollo Emprendedor, América Latina Y La Experiencia Internacional con la colaboración de, Pablo Angelelli y Virginia Moori Koenig. CAF (Corporación Andina de Fomento).

- Dirección web: http://www.sinergiacolombia.org/003porque.htm. 